\title{
Evaluation of an Indirect-ELISA Test for Trypanosoma evansi Infection (Surra) in Buffaloes and Its Application to a Serological Survey in Thailand
}

\author{
Arthur Kocher, ${ }^{1,2}$ Marc Desquesnes, ${ }^{1,2}$ Ketsarin Kamyingkird, ${ }^{2}$ Sarawut Yangtara, \\ Emilye Leboucher, ${ }^{1,2}$ Pranee Rodtian, ${ }^{3}$ Alan Dargantes, ${ }^{4}$ and Sathaporn Jittapalapong ${ }^{2}$ \\ ${ }^{1}$ Centre de Coopération Internationale en Recherche Agronomique pour le Développement (CIRAD), UMR InterTryp, \\ 34398 Montpellier, France \\ ${ }^{2}$ Faculty of Veterinary Medicine, Kasetsart University, Chatuchak, Bangkok 10900, Thailand \\ ${ }^{3}$ The Fifth Regional Livestock Office, Department of Livestock Development (DLD), Chiang Mai 50300, Thailand \\ ${ }^{4}$ Central Mindanao University, 8710 Mindanao, Philippines
}

Correspondence should be addressed to Sathaporn Jittapalapong; fvetspj@ku.ac.th

Received 22 April 2015; Accepted 11 May 2015

Academic Editor: P. A. C. Maple

Copyright (C) 2015 Arthur Kocher et al. This is an open access article distributed under the Creative Commons Attribution License, which permits unrestricted use, distribution, and reproduction in any medium, provided the original work is properly cited.

\begin{abstract}
Surra, caused by Trypanosoma evansi, is a neglected disease due to frequent subclinical evolution, especially in bovines in Asia. However, acute and chronic signs are regularly observed, with significant sanitary and economic impacts. In this study, we evaluated and applied an indirect-ELISA test for the detection of anti-T. evansi immunoglobulin G in buffaloes using antibovine conjugate. Based on buffalo reference sera from the Philippines, a two-graph receiver operating characteristics analysis (TG-ROC) was conducted to define an optimal cut-off value; sensitivity and specificity were estimated at $92.5 \%$ and $94.2 \%$, respectively. A crosssectional serological survey was carried out in the major buffalo breeding areas of Thailand; 892 buffaloes from 8 provinces were sampled in North, Northeastern, and Southern Thailand. Seropositive buffaloes were found in all 8 provinces, on $20.3 \%$ of farms for an overall prevalence of $12.2 \%$ (95\% CI 10.2-14.5\%). Nearly one-third of the sampled population was exposed to infection. Broader sampling would be necessary but is not possible in the southern half-wild breeding systems. According to our results, buffaloes may constitute a large and robust reservoir for T. evansi, which is a permanent threat to other livestock such as cattle and horses as well as wild animals such as elephants in Southest Asia.
\end{abstract}

\section{Introduction}

Surra is a multispecies disease caused by Trypanosoma evansi, originating from Africa, where it mainly affects camels. It spread to Latin America and Asia, where it mostly affects horses, dogs, cattle, and buffaloes [1]. It is an underestimated and neglected disease because of the frequent subclinical evolution of the infection. This is especially the case in Asian swamp buffaloes, because, even though clinical signs are frequent at the moment of the outbreak, most of the animals become healthy carriers after some time and may act as reservoirs [2]. These healthy carriers may relapse into clinical infection under the pressure of stress or intercurrent diseases and thus remain permanent sources of infection for susceptible livestock, leading to enzo-epizootic situations [3]. Under these conditions, the sensitivity of parasitological tests is very low and it is difficult to establish a clear diagnosis. Consequently, assessing the sanitary and economic impacts of surra is even more challenging. However, recent studies based on population modelling demonstrated a highly significant impact of surra in buffaloes in the Philippines [4]. Other studies demonstrated the interference of surra in vaccination against Foot and Mouth Disease (FMD) [5, 6] and haemorrhagic septicaemia (HS) [7, 8], as suggested from field observations [9]. In the context of a large-scale control campaign against FMD and HS in Southeast Asia where surra is enzootic, this draws fresh attention to the disease. 
Antibody detection using indirect-ELISA with soluble antigens of T. evansi from whole cell lysate is recommended by the OIE [10] and has been used for a long time [11]. Standardization and validation methods have been developed [12-15]. ELISA T. evansi, initially used on camels [16], has been adapted to other species such as horses [17, 18], buffaloes [19], cattle [20], and pigs [21]. However, the local development of serological tests, including parasite production, mean population responses, and selection of control sera is necessary to cope with the variability in response that may exist between parasite stocks and hosts from different geographic areas $[13,22]$.

A survey carried out in Thai dairy cattle demonstrated the presence of $T$. evansi infection in 12 out of the 13 provinces investigated and showed that around $25 \%$ of the cattle population was exposed to the infection [23]. Little is known on the potential impact of surra in the Thai buffalo population which was estimated at around 1.4 million head in 2009. Here, we standardized and evaluated an indirect-ELISA test for the detection of anti-T. evansi immunoglobulins $G$ (IgG) in buffaloes. The test was then applied to a serological survey in the main buffalo-breeding areas of Thailand.

\section{Materials and Methods}

\subsection{Indirect-ELISA T. evansi Standardization}

2.1.1. Protocol. The preparation of soluble antigens from a T. evansi strain isolated in Thailand and ELISA procedures were derived from a study previously carried out in dairy cattle in Thailand [23].

In preliminary studies, performance of ELISA in buffaloes was compared between Protein A and antibovine conjugates. The latest provided a higher contrast between samples from infected and noninfected animals. Consequently, antibovine conjugate was used in the present study.

Briefly, Microtest 96-well Polysorp Nunc immunoplates (Nunc, Roskilde, Denmark) were coated with $100 \mu \mathrm{L} /$ well of $T$. evansi soluble antigen $(5 \mu \mathrm{g} / \mathrm{mL})$ in carbonate buffer (0.05 M, pH 9.6) and incubated overnight at $4^{\circ} \mathrm{C}$. The plates were blocked with $150 \mu \mathrm{L} /$ well of blocking buffer (BB) (Phosphate Saline Buffer (PBS), 0.1\% Tween 20 (Labchem, Pittsburg, USA), 7\% skim milk powder (ref.: 190-12865, Wako Pure Chemical Industries Ltd., Osaka, Japan)) with permanent shaking $(150 \mathrm{rpm})$ for $30 \mathrm{~min}$ at $37^{\circ} \mathrm{C}$. The $\mathrm{BB}$ was discarded. Sera diluted 1:100 in BB were transferred in duplicate to the ELISA plates. After incubating, under the same conditions as previously, the plates were washed eight times with washing buffer (WB) (PBS, 0.1\% Tween 20). Then, $100 \mu \mathrm{L}$ of peroxidase-conjugated antibovine IgG (ref: A5295, Sigma-Aldrich, USA), diluted 1:25,000 in BB, was added and the plates incubated for $30 \mathrm{~min}$ at $37^{\circ} \mathrm{C}$ with shaking $(150 \mathrm{rpm})$. After washing eight times with $\mathrm{WB}, 100 \mu \mathrm{L}$ of the substrate/chromogen complex $3,3^{\prime}, 5,5^{\prime}$ tetramethylbenzidine (TMB) (SureBlue TMB, KPL, Maryland, USA) was added. The plates were incubated, without shaking, in a dark room for $30 \mathrm{~min}$. Optical density (OD) was measured at $620 \mathrm{~nm}$ in an ELISA reader (Dynex Technologies, VA, USA).
2.1.2. Selection of Controls and Definition of a Relative Percentage of Positivity. Our stock of reference serum samples from infected and noninfected buffaloes being from the Philippines and in limited amount, it was used only to determine the cut off value and the test's performances (see Section 2.1.3).

To be representative of the target population, local control serum was selected from Thai buffalo samples. A first batch of buffalo sera from Northeastern Thailand was analysed by ELISA and the results expressed as optical density (OD). Arbitrary and temporary low and high cut-off values (COVl $\&$ COVh) were used in order to define negative and positive samples, respectively. A farm was considered as noninfected if all samples exhibited OD $<$ COVl. A pool of negative samples was made with samples from noninfected farms. A pool of positive samples was made with samples exhibiting OD > COVh. The mean OD of negative and positive samples ( $\mu \mathrm{n}$ and $\mu \mathrm{p}$ ) were computed. Three negative controls $(\mathrm{C}-$ ) and three positive controls $(\mathrm{C}+)$ (low, medium, and high responses), representative of noninfected and infected Thai buffalo populations were selected as follows:

$$
\begin{aligned}
& \mathrm{C} 1-\approx 0.9 \times \mu \mathrm{n}, \\
& \mathrm{C} 2-\approx \mu \mathrm{n}, \\
& \mathrm{C} 3-\approx 1.1 \times \mu \mathrm{n}, \\
& \mathrm{C} 1+\approx 0.9 \times \mu \mathrm{p}, \\
& \mathrm{C} 2+\approx \mu \mathrm{p}, \\
& \mathrm{C} 3+\approx 1.1 \times \mu \mathrm{p} .
\end{aligned}
$$

Afterwards, ELISAs were run for all samples (reference and survey) in duplicate with the three $\mathrm{C}+$, the three $\mathrm{C}-$, and 2 blank wells on each plate. The blank OD value was systematically subtracted from the average OD of each sample and the results were expressed as a relative percentage of positivity (RPP) [13] as follows:

$$
\mathrm{RPP} \text { sample }=\frac{\text { mean OD sample }- \text { mean OD of } \mathrm{C}-}{\text { mean OD of } \mathrm{C}+- \text { mean OD of } \mathrm{C}-} .
$$

2.1.3. Selection of the Cut-Off Value and Evaluation of the Test. Reference sera were collected from buffaloes from the Philippines (Central Mindanao University). Forty-one adult buffaloes positive for T. evansi using Hematocrit Centrifuge technique (HCT [24]) or mouse inoculation technique (MIT) were used as positive reference samples. Thirty-eight buffaloes native and bred in an area where surra has never been detected were used as negative reference samples. All were negative with HCT, MIT, and the Card agglunitation test for trypanosomiasis (CATT/T. evansi, [25]).

The indirect-ELISA test was performed on these samples. The normal distribution of their RPP values was checked with a Shapiro-Wilk test using the stats package in $R$. To optimize the cut-off value, we performed a two-graph receiver operating characteristics analysis (TG-ROC, [26]) using the DiagnosisMed package in $R$. The misclassification cost term method was used [27] with an a priori prevalence of 


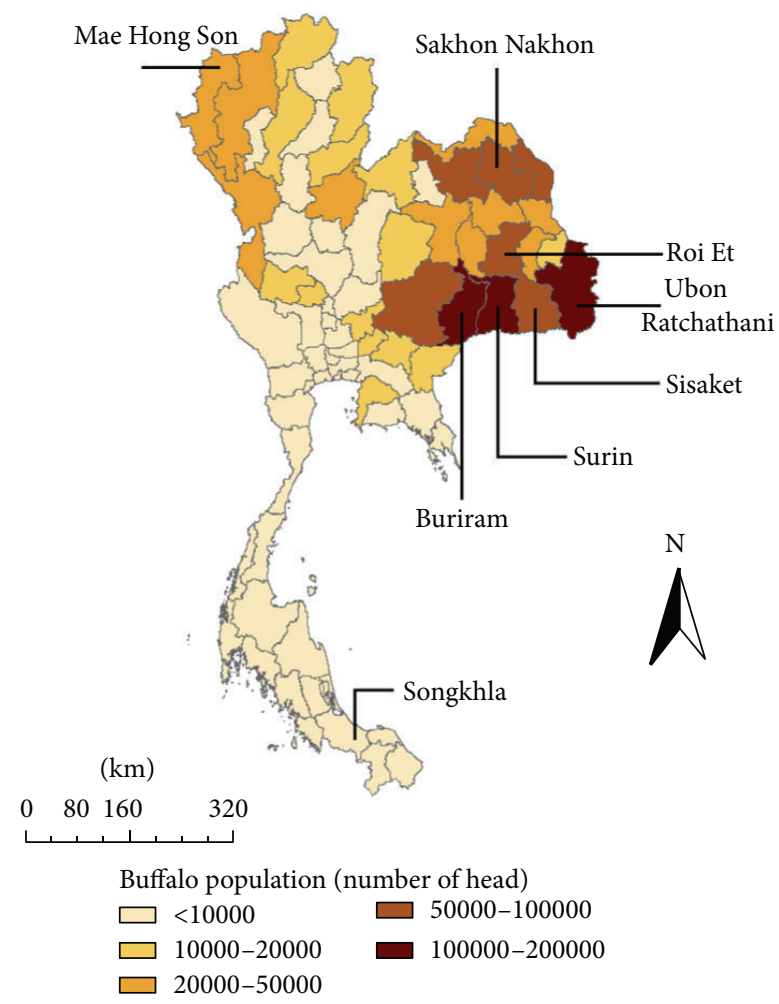

FIGURE 1: Distribution of buffalo population in Thailand (source: DLD, 2009) and sampling area. Only the names of the provinces sampled are indicated on the map.

0.2 , and a misclassification cost term of 2 . Resulting sensitivity (Se) and specificity (Sp) were computed with $95 \%$ confidence intervals.

2.2. Survey Area and Sampling. Farms were selected randomly among volunteer breeders after they were informed by the Department of Livestock Development (DLD) officers. The study area comprised eight provinces: (i) six provinces of Northeastern Thailand, with the highest buffalo population and where animals are bred in small units (2-4 heads), (ii) one province of Northern Thailand, characterized by very close breeding conditions for cattle and buffaloes, and (iii) one province of Southern Thailand, mostly characterized by a population of medium-sized herds of domesticated buffaloes, and a large population of extensive herds (almost wild animals). These provinces hold 635,000 heads, thus approximately $46 \%$ of Thai buffaloes (DLD, 1984-2009; Figure 1, Table 1).

Blood samples were collected in plain and EDTA tubes by jugular venipuncture. When immediate laboratory work was possible, the EDTA tubes were placed inside a cool box pending blood centrifugation in dry capillary tubes (Hirschmann Laborgeräte, Eberstadt, Germany) for packed cell volume (PCV) measurement and microscopic examination for the detection of trypanosomes (HCT, [24]). Blood collected in plain tubes was allowed to clot for 1-4 hours at ambient temperature and then stored at $1-4^{\circ} \mathrm{C}$ for 24 hours until the serum was separated by centrifugation (500 g) and collected for serological tests.

2.3. Data Analysis. Prevalence estimations and confidence intervals computations were made using the epitools webserver (http://epitools.ausvet.com/; [28]). Apparent prevalence values were inferred directly from the indirect-ELISA results and the Wilson binomial approximation [29] was used to compute the confidence limits. True prevalence values and confidence intervals were estimated based on the indirectELISA results and the quality of the test (Se and $\mathrm{Sp}$ ), as previously assessed, using Blaker's method [30]. An infected farm was defined as a farm in which at least one buffalo was positive. The prevalence of infected farms (PF) and the mean prevalence in infected farms $(\mathrm{PiF})$ were computed. All statistical tests were made using $R$.

\section{Results}

3.1. ELISA T. evansi Standardization. From the first batch of samples from Thailand that were tested in ELISA, a pool of 526 samples was constituted from farms considered as noninfected (all samples exhibiting OD $<0.150$ ). Their mean $\mathrm{OD}$ was 0.062 . The 3 selected negative controls (C1-, C2-, and C3-) exhibited OD values of $0.054,0.062$, and 0.066 , respectively.

Another pool of 16 positive samples was made with samples exhibiting an $\mathrm{OD}>0.350$; their mean $\mathrm{OD}$ was 0.401 . The 3 selected positive controls $(\mathrm{C} 1+, \mathrm{C} 2+$, and $\mathrm{C} 3+)$ exhibited OD values of $0.361,0.401$, and 0.442 , respectively.

From that point, ELISA was carried out using these 6 controls and results were expressed in RPP. Negative reference sera from the Philippines showed RPP values between $-7.3 \%$ and $27.2 \%$. Positive reference sera showed RPP values between $9.3 \%$ and $103.3 \%$. Their distribution is presented in Figure 2. A parametric simulation was used for the TG-ROC analysis after checking for the normality of the data with the Shapiro-Wilk test ( $p=0.0012$ and $p=0.0021$ for the positive and negative reference sera, resp.). The TG-ROC curve of the test is presented in Figure 3. The best RPP cut-off value was determined to be of $18.38 \%$, resulting in Se $=92.51 \%$ (95\% CI $84.45-100 \%)$ and $\mathrm{Sp}=94.22 \%$ (95\% CI 86.80-100\%).

3.2. Survey Sampling. In total, 892 local swamp buffaloes were sampled on 324 farms. Some $(N=697)$ were owned by small-holders from Northeastern Thailand, and the others originated from medium-holders from Southern Thailand $(N=95)$ and Northern Thailand $(N=100)$. Samples were collected in three of the four regions throughout 8 provinces: Sakon Nakhon, Roi Et, Surin, Ubon Ratchathani, Sisaket, Buriram, Maehongson, and Songkhla. Out of 788 individuals for which information was available, the animals were aged between 3 months and 20 years, with a mean of 4.9 years (95\% CI \pm 0.24 years); 66.1\% (95\% CI \pm 3.3 years) were aged 5 years or under, while "late career" animals aged 14 years and over accounted for only $3.4 \%$ ( $95 \% \mathrm{CI} \pm 1.27$ years) of the population. Sex was recorded for 817 animals. A strong predominance of females was observed: $84 \%$ (95\% CI $\pm 2.56 \%$ ). 
TABLE 1: Results of the buffalo serological survey by indirect-ELISA in 8 provinces of Thailand.

\begin{tabular}{|c|c|c|c|c|c|c|c|c|}
\hline Region & Provinces & Census 2009 & $n$ & $n^{+}$ & $\begin{array}{c}\mathrm{AP}(\%) \\
(95 \% \mathrm{CI})\end{array}$ & $\begin{array}{c}\mathrm{TP}(\%) \\
(95 \% \mathrm{CI})\end{array}$ & PF (\%) & $\operatorname{PiF}(\%)$ \\
\hline North & Maehongson & 23,761 & 100 & 18 & $\begin{array}{c}18 \\
(11.7-26.7)\end{array}$ & $\begin{array}{c}13.6 \\
(6-23.4)\end{array}$ & 38.1 & 39.2 \\
\hline \multirow{6}{*}{ Northeast } & Buriram & 105,177 & 138 & 17 & $\begin{array}{c}12.3 \\
(7.8-18.8)\end{array}$ & $\begin{array}{c}7.3 \\
(1.9-14.7)\end{array}$ & 30.4 & 44.9 \\
\hline & Roi Et & 70,23 & 81 & 11 & $\begin{array}{c}13.6 \\
(7.8-22.7)\end{array}$ & $\begin{array}{c}\mathbf{8 . 7} \\
(1.7-18.7)\end{array}$ & 17.1 & 64.3 \\
\hline & Sakon Nakhon & 75,647 & 208 & 27 & $\begin{array}{c}13.0 \\
(9.1-18.2)\end{array}$ & $\begin{array}{c}\mathbf{8} \\
(3.5-13.8)\end{array}$ & 18 & 79.7 \\
\hline & Sisaket & 98,427 & 59 & 1 & $\begin{array}{c}1.7 \\
(0.3-9)\end{array}$ & $\begin{array}{c}\mathbf{0} \\
(0-3.3)\end{array}$ & 4.5 & $100.0(1$ infected $)$ \\
\hline & Surin & 120,886 & 73 & 7 & $\begin{array}{c}9.6 \\
(4.7-18.5)\end{array}$ & $\begin{array}{c}4.2 \\
(0-14.1)\end{array}$ & 25 & 45.7 \\
\hline & Ubon Ratcha. & 136,528 & 138 & 6 & $\begin{array}{c}4.3 \\
(2-9.2)\end{array}$ & $\begin{array}{c}\mathbf{0} \\
(0-7.6)\end{array}$ & 10.8 & 47.2 \\
\hline South & Songkhla & 4,431 & 95 & 22 & $\begin{array}{c}23.2 \\
(15.8-32.6)\end{array}$ & $\begin{array}{c}19.4 \\
(10.8-29.8)\end{array}$ & 71.4 & 29.2 \\
\hline Total & & 635,087 & 892 & 109 & $\begin{array}{c}12.2 \\
(10.2-14.5)\end{array}$ & $\begin{array}{c}7.2 \\
(4.9-9.8)\end{array}$ & 20.3 & 55.7 \\
\hline
\end{tabular}

$n$ : number of serum samples tested. $n^{+}$: number of seropositive samples by indirect-ELISA. CI: confidence interval. AP: apparent seroprevalence rate per province $\left(\mathrm{AP}=n^{+} / n\right)$. TP: true prevalence estimates. PF: percentage of infected farms (at least one seropositive sample). PiF: mean seroprevalence rate on infected farms.

The majority of males were under 5 years old. Farm sizes ranged from 1 to 20 buffaloes. The vast majority of farms were small units of 1 to 3 animals $(77.7 \%, 95 \% \mathrm{CI} \pm 2.9 \%)$ while units of 10 or more head only amounted to $3.7 \%$ of farms $(95 \%$ $\mathrm{CI} \pm 1.3 \%)$ and were mostly located in the Southern Songkhla Province.

3.3. Seroprevalence Estimations. Out of 892 samples, 109 were positive, resulting in an estimated overall apparent prevalence with ELISA of $12.2 \%$ (95\% CI 10.2-14.5\%). All provinces showed serological evidence of infection. Provincial seroprevalence ranged from $1.7 \%$ (95\% CI $0.3-9 \%$ ) in Sisaket to 23.2\% (95\% CI 15.8-32.6\%) in Songkhla (Figure 4, Table 1). Estimates of true prevalence are lower than apparent prevalence in every province ranging from $0 \%(95 \%$ CI 0 3.3\%) in Sisaket to $19.4 \%$ (95\% CI 10.8-29.8\%) in Songkhla, for an overall true prevalence estimation of 7.2\% (95\% CI 4.99.8\%).

Out of 324 farms, 66 had serological evidence of infection (20.3\%): $4.5 \%$ of the farms were infected in Sisaket, $38.1 \%$ in Maehongson, and $71.4 \%$ in Songkhla (Table 1). In infected farms, the herd seroprevalence ranged from $5.0 \%$ to $100 \%$. The average herd prevalence in these infected farms (PiF) ranged from $29.2 \%$ in Songkhla to $79.7 \%$ in Sakon Nakhon (the $100 \%$ in Sisaket was only due to a seropositive sample in a single animal farm), resulting in an overall mean of $55.7 \%$.

In 465 samples, the PCV values ranged from 16 to $54 \%$ with an average of $36.1 \%$ (95\% CI $\pm 0.5 \%$ ). When comparing the average PCV of seronegative animals $(36.2 \%$; $95 \% \mathrm{CI} \pm 0.5 \%)$ with that of seropositive animals (35.8\%; $95 \%$ CI $\pm 1.5 \%$ ), no significant difference was observed ( $Z$ test:

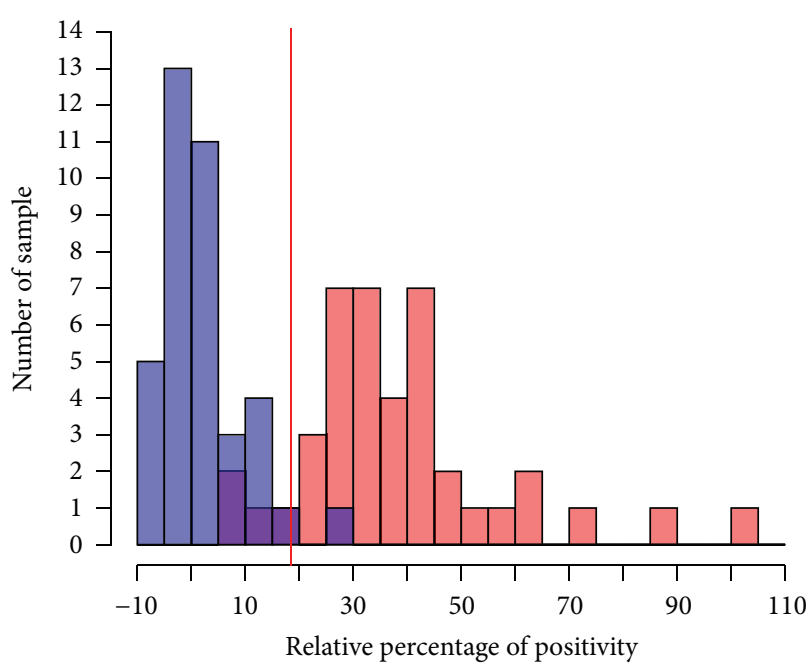

FIGURE 2: Distribution of indirect-ELISA relative percentage of positivity values in negative (blue) and positive (red) buffalo reference sera from the Philippines. The red vertical line indicates the optimal cut-off value as determined by the TG-ROC analysis.

$p=0.7)$. Eleven samples were positive for trypanosomes by microscopic examination of the buffy coat; amongst them, 9 were considered as T. theileri, based on the size and the shape of the parasite; these samples were seronegative to ELISA. Another 2 samples, identified as Trypanosoma species (suspected to be T. evansi), were seropositive to ELISA and considered as T. evansi infection. 


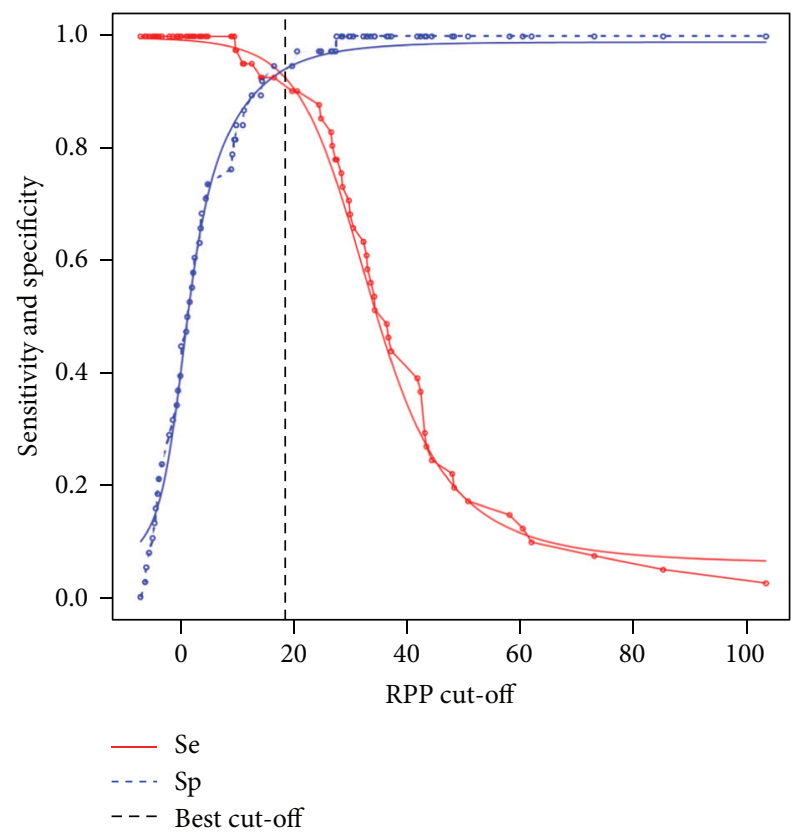

FIgURE 3: TG-ROC curves. Parametric (smooth lines) and nonparametric (dotted lines) estimates of sensitivity and specificity based on the relative percentage of positivity (RPP) cut-off. The optimal cut-off according to the misclassification cost term (MCT) criteria is indicated by a vertical line.

Animals under 1 year old were all negative. Prevalence increased with age, ranging from $4.1 \%$ ( $\leq 2$ years) to $10.8 \%(2<$ $x \leq 5$ years) and $16.8 \%$ ( $>5$ years). The seroprevalence among these three age groups was significantly different even after Bonferroni correction to account for multiple comparisons $\left(\chi^{2}=19.59, p=5.56 e-05\right)$.

Six males and 83 females tested seropositive with ELISA for a respective prevalence of $4.5 \%$ and $12 \%$, resulting in a significantly higher rate in females $\left(\chi^{2}=6.40, p=0.01\right)$.

\section{Discussion}

In all previous studies, indirect-ELISA for trypanosomes exhibited high sensitivity and specificity, generally $>90 \%$, but there is no real gold standard for serological tests in trypanosomes [10]. Previous results obtained with this ELISA T. evansi in experimentally infected cattle showed satisfactory sensitivity and specificity [31]. In the present study, the evaluation of the test on buffalo reference sera, indicates satisfying performances ( $\mathrm{Se}=92.5 \%$ and $\mathrm{Sp}=94.2 \%)$, justifying its application to a survey in Thailand. The seroprevalence rates observed in all the Thai provinces sampled showed a wide distribution of T. evansi in the country. Estimations were lower than those of three previous studies indicating seroprevalence rates of around $20 \%[2,32,33]$, as well as those of recent surveys performed in Vietnam [34, 35], but they are close to those recently observed in Thai dairy cattle [23]. Higher values were estimated in the provinces of the North and South compared to the Northeast. The estimations of infected farm rates were relatively high. In our sample, around $30 \%(273 / 892)$ of the animals sampled were living in an infected farm, which suggests that one third of the explored population may be exposed to infection.

Higher seroprevalence rates were observed in older animals. No seropositive samples were detected among animals under 1 year old, most probably because of the limited exposure of young animals to insects and the role of adults acting as a screen protecting young animals [36, 37]. The higher seroprevalence rates observed among females compared to males need to be viewed with caution. Indeed, as the male population was clearly younger than the female population, probably because of the smaller number of male reproducers, this can introduce confusing bias. However, other physiological or behavioural risk factors related to sex cannot be totally excluded with these results.

Overall, few HCT were positive since only 2 samples were suspected to show T. evansi, which confirms the lack of sensitivity of this method, especially in host species able to maintain low parasitaemia, such as buffaloes [38]. When T. theileri was observed (9 samples), ELISA was negative, indicating the absence of cross reaction.

No statistical difference was observed between the haematocrit values of seropositive and seronegative animals. As anaemia is one of the main symptoms of surra, this suggests that buffaloes have a relative tolerance to the parasite, as previously described [1]. Yet, this statement needs to be tempered by other results, such as those of a cross-sectional study in the Philippines [4], showing lower fertility and higher mortality rates among buffaloes in areas of high $T$. evansi seroprevalence. Nevertheless, asymptomatic infections among buffaloes are probably frequent, which allows them to act as a robust and efficient reservoir for T. evansi, therefore representing a risk for other livestock, such as cattle and horses. The latter are highly sensitive to the parasite, with the infection frequently leading to death [1]. Consequently, in Southeast Asia, it is highly recommended to keep horses separate from cattle and buffaloes [3].

\section{Conclusion}

Diagnosis is an essential prerequisite in disease investigation. This indirect-ELISA using soluble antigens from whole cell lysate of $T$. evansi and antibovine conjugate was proved to have adequate qualities for serodiagnosis in buffaloes. ELISA is particularly adapted for screening of surra in host species such as buffaloes which tends to maintain low parasitemia [38], making difficult the detection of the parasite with direct methods such as HCT. ELISA providing results on a continuous scale, its positivity cut-off can be adapted to various contexts and objectives [13]. Lyophilisation of the trypanosome soluble antigens for ELISA is currently under validation in a Twinning Programme carried out by CIRAD (Centre de Coopération Internationale en Recherche Agronomique pour le Développement, Montpellier, France) and CIRDES (Centre International de Recherche Développement de l'Elevage en zone Sub-humide, BoboDioulasso, Burkina Faso) under the umbrella of the OIE (Office International des Epizooties, Paris, France). Once 


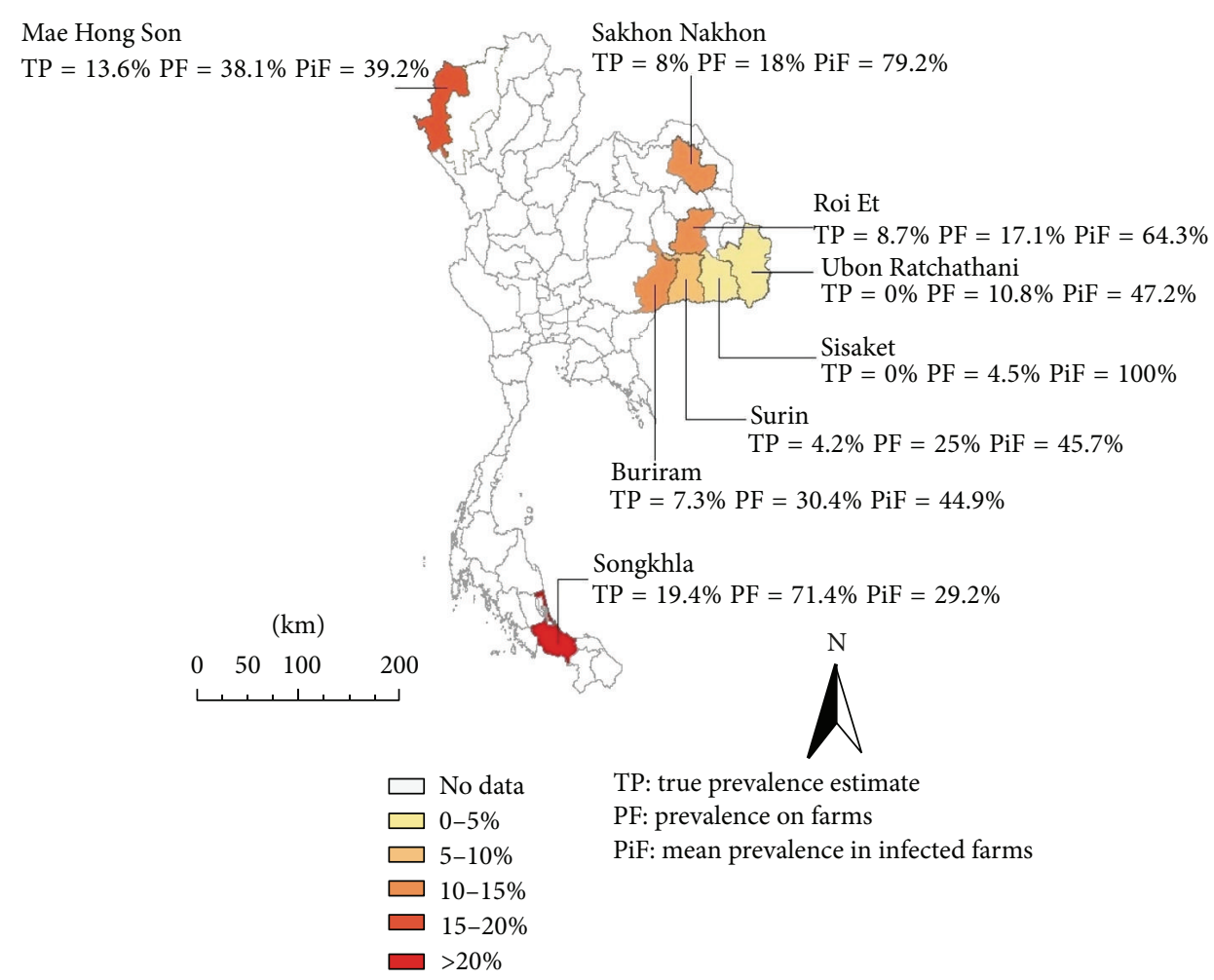

FIGURE 4: Geographical representation of the serological survey results using indirect-ELISA in buffalo farms in Thailand.

the "lyophilized antigens" would be validated, ELISA T. evansi could be more easily distributed for general validation and use in Southeast Asian countries, especially Cambodia, Vietnam, and Laos, for which little information is available on the prevalence of T. evansi infection. Application of this ELISA could help us to evaluate the potential impact of surra in Southeast Asian livestock, especially if the models recently developed in the Philippines [4] can be adapted to other countries and host-species. Decision treatments of animals can be systematic or based on the observation of clinical signs and/or parasitological and/or serological results, with various benefits that could be evaluated as described by Dargantes et al. [4].

\section{Conflict of Interests}

The authors declare that there is no conflict of interests regarding the publication of this paper.

\section{Acknowledgments}

The authors wish to express our sincere gratitude to the Thailand International Cooperation Agency (TICA) for the financial and diplomatic support given to the project. This research was also funded by Kasetsart University Research and Development Institution (KURDI), Kasetsart University. The authors are grateful to the DLD and staff for their support in field work.

\section{References}

[1] M. Desquesnes, P. Holzmuller, D.-H. Lai, A. Dargantes, Z.R. Lun, and S. Jittaplapong, "Trypanosoma evansi and surra: a review and perspectives on origin, history, distribution, taxonomy, morphology, hosts, and pathogenic effects," BioMed Research International, vol. 2013, Article ID 194176, 22 pages, 2013.

[2] K. F. Löhr, S. Pohlpark, L. Srikitjakarn, P. Thaboran, G. Bettermann, and C. Staak, "Trypanosoma evansi infection in buffaloes in north-east Thailand. I. Field investigations," Tropical Animal Health and Production, vol. 17, no. 2, pp. 121-125, 1985.

[3] M. Desquesnes, A. Dargantes, D.-H. Lai, Z.-R. Lun, P. Holzmuller, and S. Jittapalapong, "Trypanosoma evansi and surra: a review and perspectives on transmission, epidemiology and control, impact, and zoonotic aspects," BioMed Research International, vol. 2013, Article ID 321237, 20 pages, 2013.

[4] A. P. Dargantes, R. T. Mercado, R. J. Dobson, and S. A. Reid, "Estimating the impact of Trypanosoma evansi infection (surra) on buffalo population dynamics in southern Philippines using data from cross-sectional surveys," International Journal for Parasitology, vol. 39, no. 10, pp. 1109-1114, 2009.

[5] D. N. Onah, J. Hopkins, and A. G. Luckins, "Effects of Trypanosoma evansi on the output of cells from a lymph node draining the site of Pasteurella haemolytica vaccine administration," Journal of Comparative Pathology, vol. 117, no. 1, pp. 73-82, 1997.

[6] S. Shahsavandi, M. Salehizadeh, K. Esmail-Nia, and M. M. Ebrahimi, "Evaluation of humoral immune response to footand-mouth-disease vaccination in experimentally infected 
guinea pigs with Trypanosoma evansi," Archives of Razi Institute, vol. 52, no. 9, p. 1, 2001.

[7] W. G. Holland, L. N. My, T. V. Dung et al., "The influence of T. evansi infection on the immuno-responsiveness of experimentally infected water buffaloes," Veterinary Parasitology, vol. 102, no. 3, pp. 225-234, 2001.

[8] L. D. Singla, P. D. Juyal, and N. S. Sharma, "Immune responses to haemorrhagic septicaemia (HS) vaccination in Trypanosoma evansi infected buffalo-calves," Tropical Animal Health and Production, vol. 42, no. 4, pp. 589-595, 2010.

[9] L. Touratier, “The Office International des epizooties ad hoc group on non tsetse animal trypanosomoses: its origin, scope and perspectives," Memórias do Instituto Oswaldo Cruz, vol. 94, no. 2, pp. 191-194, 1999.

[10] OIE, "Chapter 2.1.17: Trypanosoma evansi infection (Surra)," in Terrestrial Manual, vol. 2012, pp. 1-14, World Organisation for Animal Health, OIE, Paris, France, 2012.

[11] A. G. Luckins, "Detection of antibodies in Trypanosomeinfected cattle by means of a microplate enzyme-linked immunosorbent assay," Tropical Animal Health and Production, vol. 9, no. 1, pp. 53-62, 1977.

[12] R. Bocquentin and G. Duvallet, "Amélioration de la reproductibilité du test ELISA adapté à la détection d'anticorps antiTrypanosoma congolense chez les bovins," Revue d'Élevage et de Médecine Vétérinaire des Pays Tropicaux, vol. 43, no. 2, pp. 179186, 1990.

[13] M. Desquesnes, "Standardisations internationale et régionale des épreuves immuno-enzymatiques : méthodes, intérêts et limites," Revue Scientifique et Technique (Office International des Épizooties), vol. 16, no. 3, pp. 809-823, 1997.

[14] O. Diall, O. B. Touré, B. Diarra, and Y. Sanogo, "Trypanosomiasis and trypanocidal treatments in Ndama calves in a glossina infected area (the ranch of Madina-Diassa in Mali)," Revue d'Elevage et de Medecine Veterinaire des Pays Tropicaux, vol. 45, no. 2, pp. 155-161, 1992.

[15] P. F. Wright, E. Nilsson, E. M. Van Rooij, M. Lelenta, and M. H. Jeggo, "Standardisation and validation of enzyme-linked immunosorbent assay techniques for the detection of antibody in infectious disease diagnosis," Revue Scientifique et Technique (International Office of Epizootics), vol. 12, no. 2, pp. 435-450, 1993.

[16] E. Zweygarth, C. Sabwa, and D. Röttcher, "An enzyme-linked immunosorbent assay for the detection of antibodies to Trypanosoma (T.) brucei evansi in camels (Camelus dromedarius) using peroxidase-conjugated protein A," Tropical Medicine and Parasitology, vol. 37, no. 2, pp. 105-106, 1986.

[17] C. M. Monzon, O. A. Mancebo, and A. M. Russo, "Antibody levels by indirect ELISA test in Trypanosoma evansi infected horses following treatment with quinapyramine sulphate," Veterinary Parasitology, vol. 111, no. 1, pp. 59-63, 2003.

[18] A. Reyna-Bello, F. A. García, M. Rivera, B. Sansó, and P. M. Aso, "Enzyme-linked immunosorbent assay (ELISA) for detection of anti-Trypanosoma evansi equine antibodies," Veterinary Parasitology, vol. 80, no. 2, pp. 149-157, 1998.

[19] W. G. Holland, N. G. Thanh, L. N. My, T. T. Do, B. M. Goddeeris, and J. Vercruysse, "Prevalence of Trypanosoma evansi in water buffaloes in remote areas in Northern Vietnam using PCR and serological methods," Tropical Animal Health and Production, vol. 36, no. 1, pp. 45-48, 2004.

[20] S. A. Reid and D. B. Copeman, "Evaluation of an antibodyELISA using five crude antigen preparations for the diagnosis of
Trypanosoma evansi infection in cattle," Veterinary Parasitology, vol. 104, no. 1, pp. 79-84, 2002.

[21] W. G. Holland, N. G. Thanh, T. T. Do, S. Sangmaneedet, B. Goddeeris, and J. Vercruysse, "Evaluation of diagnostic tests for Trypanosoma evansi in experimentally infected pigs and subsequent use in field surveys in North Vietnam and Thailand," Tropical Animal Health and Production, vol. 37, no. 6, pp. 457467, 2005.

[22] M. Greiner, C. R. Franke, D. Bohning, and P. Schlattmann, "Construction of an intrinsic cut-off value for the seroepidemiological study of Trypanosoma evansi infections in a canine population in Brazil: a new approach towards an unbiased estimation of prevalence," Acta Tropica, vol. 56, no. 1, pp. 97-109, 1994.

[23] M. Desquesnes, K. Kamyingkird, M. Pruvot et al., "AntibodyELISA for Trypanosoma evansi: application in a serological survey of dairy cattle, Thailand, and validation of a locally produced antigen," Preventive Veterinary Medicine, vol. 90, no. 3-4, pp. 233-241, 2009.

[24] P. T. Woo, "The haematocrit centrifuge technique for the diagnosis of African trypanosomiasis," Acta Tropica, vol. 27, no. 4, pp. 384-386, 1970.

[25] E. B. Songa and R. Hamers, "A card agglutination test (CATT) for veterinary use based on an early VAT RoTat 1/2 of Trypanosoma evansi," Annales de la Societe Belge de Medecine Tropicale, vol. 68, no. 3, pp. 233-240, 1988.

[26] M. Greiner, D. Sohr, and P. Göbel, "A modified ROC analysis for the selection of cut-off values and the definition of intermediate results of serodiagnostic tests," Journal of Immunological Methods, vol. 185, no. 1, pp. 123-132, 1995.

[27] M. Greiner, "Two-graph receiver operating characteristic (TGROC): update version supports optimisation of cut-off values that minimise overall misclassification costs," Journal of Immunological Methods, vol. 191, no. 1, pp. 93-94, 1996.

[28] E. S. G. Sergeant, Epitools Epidemiological Calculators, AusVet Animal Health Services, Australian Biosecurity Cooperative Research Center for Emerging Infectious Diseases, 2009.

[29] L. D. Brown, T. T. Cai, and A. DasGupta, "Interval estimation for a binomial proportion," Statistical Science, vol. 16, no. 2, pp. 101-117, 2001.

[30] J. Reiczigel, J. Földi, and L. Ózsvári, "Exact confidence limits for prevalence of a disease with an imperfect diagnostic test," Epidemiology and Infection, vol. 138, no. 11, pp. 1674-1678, 2010.

[31] M. Desquesnes, K. Kamyingkird, T. Vergne, N. Sarataphan, R. Pranee, and S. Jittapalapong, "An evaluation of melarsomine hydrochloride efficacy for parasitological cure in experimental infection of dairy cattle with Trypanosoma evansi in Thailand," Parasitology, vol. 138, no. 9, pp. 1134-1142, 2011.

[32] A. Kashemsant, M. Pholpark, S. Pholpark, S. Srihakim, and K. Leidl, "Epidemiological pattern of Trypanosoma evansi in Northeast Thailand and control measures," Journal of the Thai Veterinary Medicine Association, vol. 40, no. 3-4, pp. 84-92, 1989.

[33] D. Tuntasuvan and A. Luckins, "Status of Surra in livestock in Thailand," Journal of Protozoological Research, vol. 8, pp. 162170, 1998.

[34] Q. D. Nguyen, T.-T. Nguyen, Q. P. Pham, N. M. Le, G. T. T. Nguyen, and N. Inoue, "Seroprevalence of Trypanosoma evansiinfection in water buffaloes from the mountainous region of north vietnam and effectiveness of trypanocidal drug treatment," Journal of Veterinary Medical Science, vol. 75, no. 9, pp. 1267-1269, 2013. 
[35] T.-T. Nguyen, M. Zhou, N. Ruttayaporn et al., "Diagnostic value of the recombinant tandem repeat antigen TeGM6-4r for surra in water buffaloes," Veterinary Parasitology, vol. 201, no. 1-2, pp. 18-23, 2014.

[36] A. T. M. Barros and L. D. Foil, "The influence of distance on movement of tabanids (Diptera: Tabanidae) between horses," Veterinary Parasitology, vol. 144, no. 3-4, pp. 380-384, 2007.

[37] L. Foil, D. Stage, W. V. Adams Jr., and C. J. Issel, "Observations of tabanid feeding on mares and foals," American Journal of Veterinary Research, vol. 46, no. 5, pp. 1111-1113, 1985.

[38] H. C. Davison, M. V. Thrusfield, A. Husein et al., "The occurrence of Trypanosoma evansi in buffaloes in Indonesia, estimated using various diagnostic tests," Epidemiology and Infection, vol. 124, no. 1, pp. 163-172, 2000. 


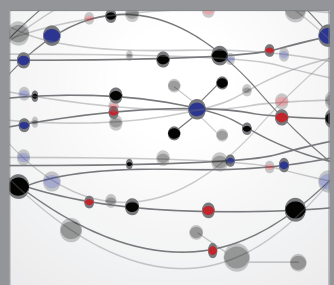

The Scientific World Journal
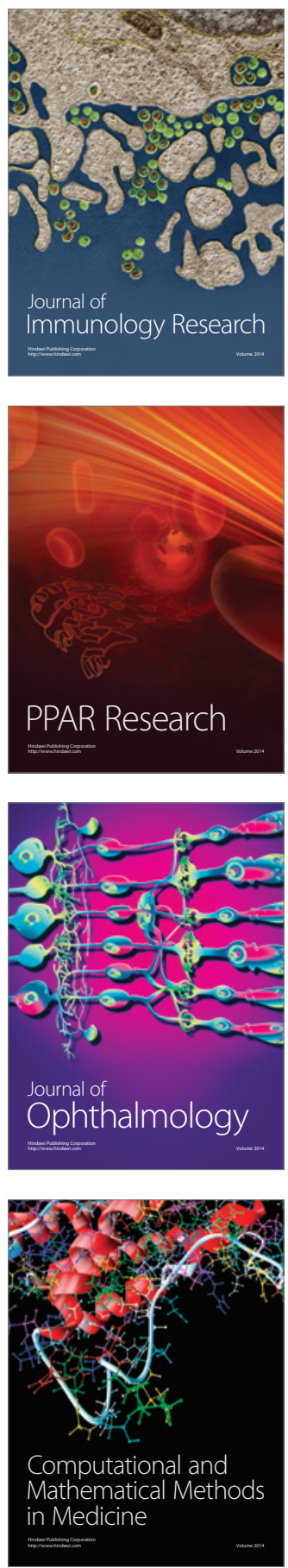

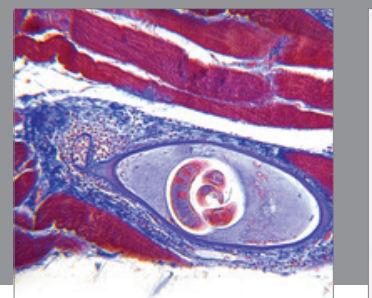

Gastroenterology

Research and Practice
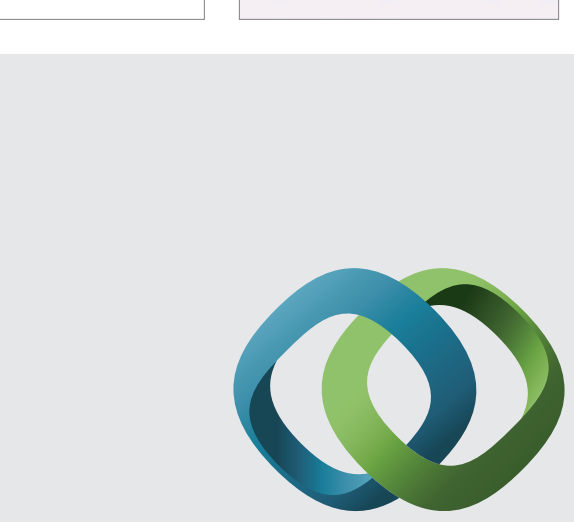

\section{Hindawi}

Submit your manuscripts at

http://www.hindawi.com
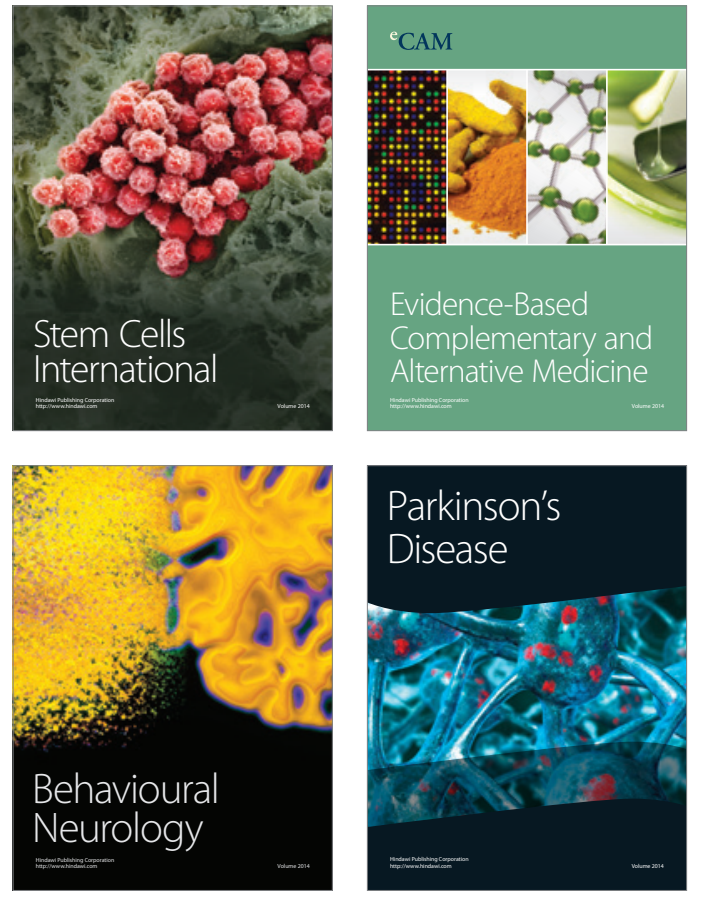
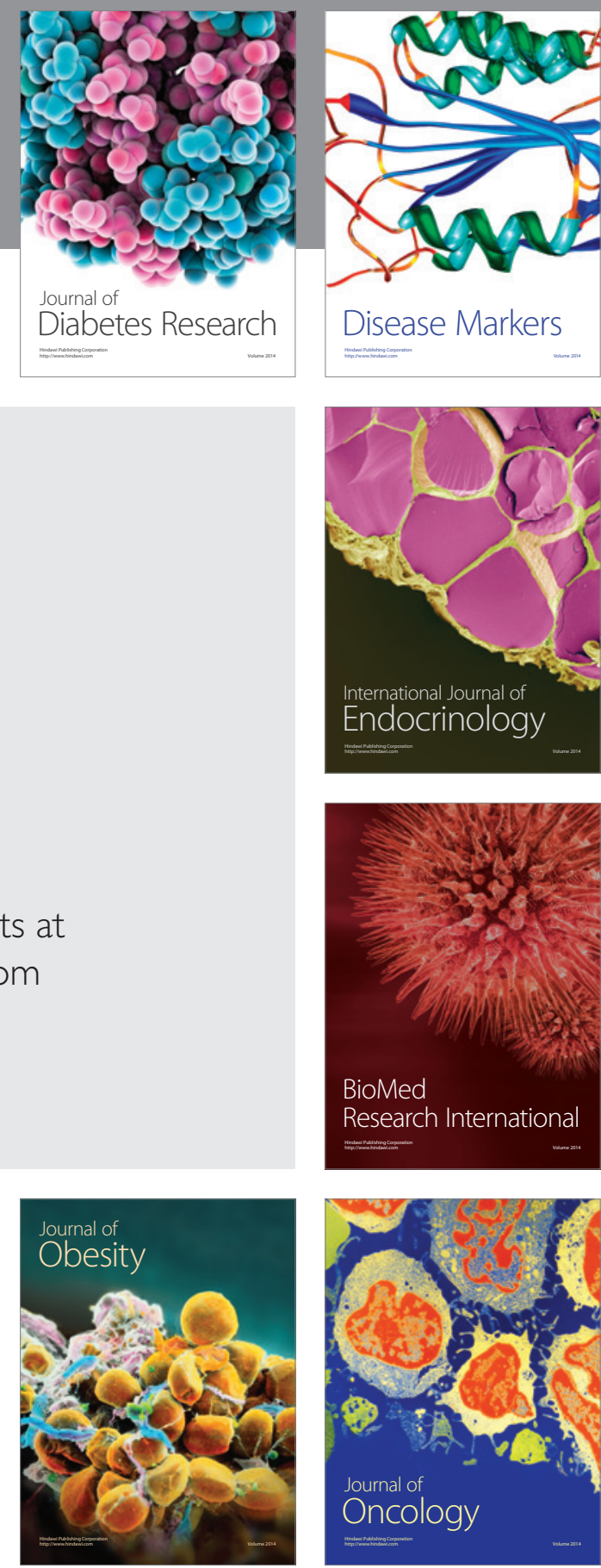

Disease Markers
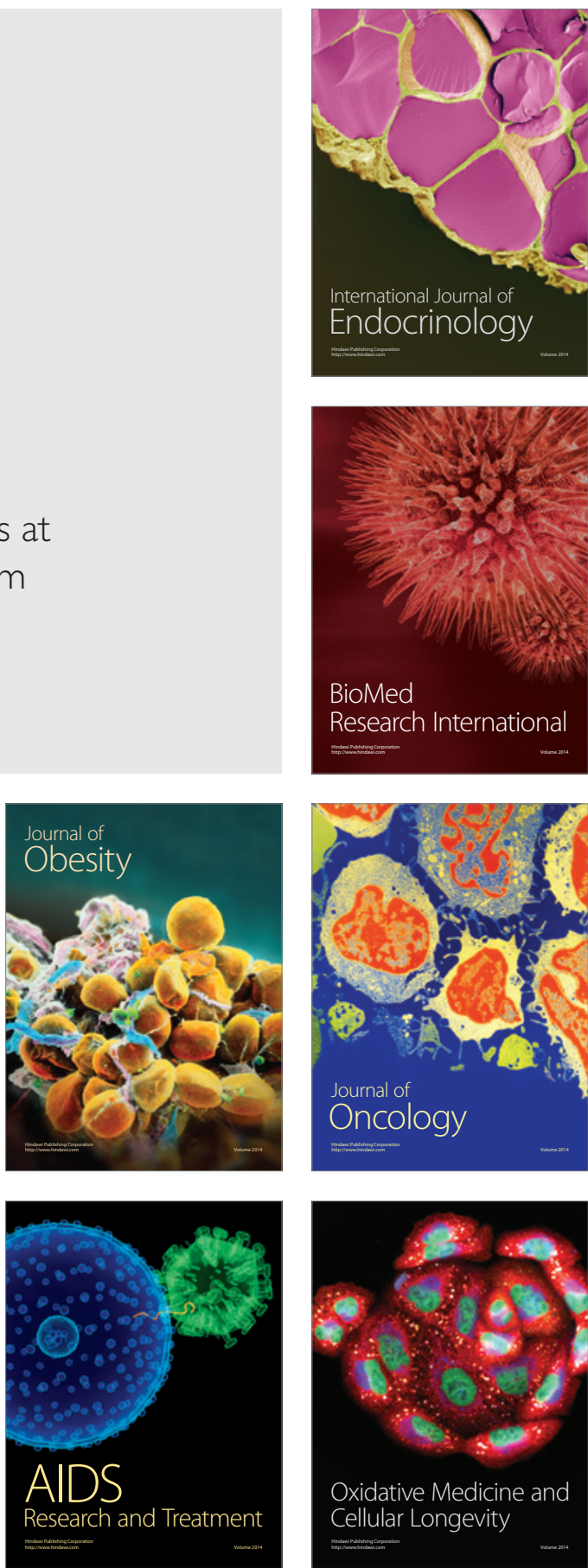